\title{
Dificuldades dos enfermeiros no atendimento ao pré-natal de risco habitual e seu impacto no indicador de morbimortalidade materno-neonatal
}

\author{
Difficulties of nurses in the attendance of prematurated habitual risk and its impact on \\ mother-neonatal morbimortality INDICATOR \\ Dificultades de las enfermeras en la asistencia al riesgo habitual prematurado y su \\ impacto en el indicador de morbimortalidad madre-neonatal
}

Tuanny Caroline Pereira de Santana ${ }^{1 *}$, Livia Maria da Silva ${ }^{1}$, Laise Risalva Farias Gouveia da Silva1, Leticia Monteiro Rocha ${ }^{1}$, Camilla Talita Silva Canhoto', Ana Cristina Farah Abdon da Silva ${ }^{1}$, Maria Inês Bezerra de Melo ${ }^{1}$, Mirella Raquel Romão Martins ${ }^{1}$, Sandra Cavalcanti Machado do Rego Barros $^{1}$; Karla da Silva Ramos ${ }^{1}$, Maria Celina Matias Rocha ${ }^{1}$, Claudia Roberta Selfes de Mendonça; Rubiane Gouveia de Souza e Silva ${ }^{1}$, Joanna Francyne Silva de Barros ${ }^{1}$, Josiana da Silva Gouveia ${ }^{1}$.

\section{RESUMO}

Objetivo: Avaliar o impacto do indicador de morbimortalidade materno-neonatal, através das dificuldades vivenciadas por enfermeiros no atendimento ao pré-natal de risco habitual. Métodos: Estudo do tipo revisão integrativa, de abordagem quantitativa e de caráter descritivo, onde foram identificados 04 artigos, que compuseram a amostra do estudo, a partir das bases de dados BDENF, LILACS, MEDLINE e PUBMED. Resultados: Algumas dificuldades encontradas pela equipe de enfermagem relacionadas ao pré-natal de risco habitual, foram alencadas, tais como, demora nos resultados dos exames solicitados, ausência de referência e contrarreferência, carência de recursos materiais e tecnológicos, espaço físico do posto de saúde inadequado para atendimento de qualidade, limitação dos enfermeiros na solicitação de exames e falta de trabalho em equipe, falta do desenvolvimento de capacitações teórico-práticas específicas, dentre outras. Considerações finais: Espera-se que este estudo possibilite uma reflexão crítica e incentive o desenvolvimento de outras pesquisas que aprofundem o tema em estudo, visando minimizar as taxas de morbimortalidade em seu componente materno-neonatal, por meio de ações que integram todos os níveis da atenção: promoção, prevenção e assistência à saúde da gestante e do recém-nascido.

Palavras-chave: Pré-natal, Enfermagem Materno-Infantil, Gestantes, atenção básica e qualidade da assistência à saúde.

\begin{abstract}
Objective: Evaluate the impact of the maternal-neonatal morbidity and mortality indicator, through the difficulties experienced by nurses in attending prenatal care at usual risk. Methods: This was an integrative review, with a quantitative approach and a descriptive character, in which four articles were identified, which comprised the study sample, from the databases BDENF, LILACS, MEDLINE and PUBMED. Results: Some difficulties encountered by nursing staff related to prenatal care at usual risk were delayed, such as delay in the results of the requested tests, absence of reference and counter-referral, lack of material and technological resources, physical space of the health post inadequate for quality care, limitation of nurses in requesting
\end{abstract}

${ }^{1}$ Faculdade Pernambucana de Saúde (FPS), Recife/PE. *E-mail: tuanny.caroline3@gmail.com

SUBMETIDO EM: 3/2019

ACEITO EM: 4/2019

PUBLICADO EM: 7/2019 
exams and lack of teamwork, lack of specific theoretical and practical training, among others. Final considerations: This study is expected to provide a critical reflection and encourage the development of other studies that deepen the theme under study, aiming to minimize the morbidity and mortality rates in its maternalneonatal component, through actions that integrate all levels of attention : promotion, prevention and health care of pregnant women and newborns.

Key words: Prenatal Care, Maternal-child nursing, Pregnancy Women, Primary Health Care and Quality of Health Care.

\section{RESUMEN}

Objetivo: Evaluar el impacto del indicador de morbilidad y mortalidad materno-neonatal, a través de las dificultades experimentadas por las enfermeras para asistir a la atención prenatal con riesgo habitual. Métodos: Esta fue una revisión integradora, con un enfoque cuantitativo y un carácter descriptivo, en el que se identificaron cuatro artículos, que comprendían la muestra del estudio, de las bases de datos BDENF, LILACS, MEDLINE y PUBMED. Resultados: Algunas dificultades encontradas por el personal de enfermería relacionadas con la atención prenatal a riesgo habitual se retrasaron, como la demora en los resultados de las pruebas solicitadas, la ausencia de referencias y contrarreferencia, la falta de recursos materiales y tecnológicos, el espacio físico del puesto de salud inadecuados para la atención de calidad, la limitación de las enfermeras en la solicitud de exámenes y la falta de trabajo en equipo, la falta de formación teórica y práctica específica, entre otros. Consideraciones finales: Se espera que este estudio proporcione una reflexión crítica y fomente el desarrollo de otros estudios que profundicen el tema en estudio, con el objetivo de minimizar las tasas de morbilidad y mortalidad en su componente materno-neonatal, a través de acciones que integren todos los niveles de atención: promoción, prevención y atención sanitaria de embarazadas y recién nacidos.

Palabras clave: Atención prenatal, enfermería materno-infantil, mujeres embarazadas, atención primaria de salud y calidad de la atención médica.

\section{INTRODUÇÃO}

A assistência pré-natal se faz presente em todas as Estratégias de Saúde da Família (ESF). No Brasil, em 2019 totalizou-se até agora 15.912 equipes de saúde da família (DATASUS MS, 2019), sendo reconhecida como principal porta de entrada das gestantes aos serviços de saúde de baixa, média e alta complexida, tendo como finalidade ações preventivas, curarativas ou de controle precoce de doenças, evitando complicações durante a gestação, parto e puerpério. Propondo garantir a saúde materna e fetal de qualidade e consequentemente reduzir os índices de morbimortalidade nessa população (DOMINGUES RMSM et al., 2015), além de manter o foco na universalidade, integralidade e equidade das ações. (COSTA et al., 2016).

Desde 2004 que muitos esforços vêm sendo implementados mundialmente com objetivo de proporcionar acesso universal e de qualidade de atenção à saúde ginecológica, reprodutiva e obstétrica (GAMA SGN et al., 2004).

Nos países com alta tecnologia, mais de $95,0 \%$ das gestantes realizam pelo menos uma consulta de prénatal, enquanto nos emergentes, como Colômbia e África do Sul, esse indicador de proteção contra diversas complicações que podem ocorrer no período antes do parto, atinge $62,0 \%$ e $69,0 \%$, respectivamente. No Brasil, desde 2010 a cobertura de pré-natal alcançou um percentual de $98 \%$, representando um avanço bastante relevante para a saúde pública (ROSA CQ et al., 2014).

Entretanto, percebe-se o menor acesso à assistência pré-natal de mulheres jovens, com idade entre $10 \mathrm{e}$ 19 anos, ou acima de 35 anos, caracterizando os extremos de idade, correspondendo a um risco de morte quatro vezes maior em relação as gestantes de outras idades, como também indígenas, pretas, menor escolaridade, baixa renda familiar, maior número de gestações (AQUINO PT et al, 2018) e residentes nas 
regiões Norte e Nordeste, evidenciando a persistência de desigualdades sociais quanto ao acesso aos serviços de saúde no Brasil (DOMINGUES RMSM et al., 2015).

Sabe-se que o pré-natal realizado de maneira adequada promove a detecção e a intervenção de forma antecipada das diversas situações de risco da mãe e do bebê (BRASIL MS, 2012). A intervenção e a detecção precoce de situações de risco, regulação de leitos obstétricos, assistência qualificada ao parto e ao nascimento saudável, são alguns dos determinantes de saúde, relacionados a redução as principais causas de mortalidade materna-neonatal (BRASIL MS, 2012).

Desta forma a assistência no pré-natal é adotada como política pública para reduzir o impacto desse indicador de saúde (DUARTE SJH, 2014), porém só em 2004 que o ministério da saúde instituiu a Política Nacional de Atenção Integral à Saúde da Mulher, com ênfase na melhoria a atenção obstétrica (BRASIL MS, 2004).

Outro fator importante, relacionado ao indicador de saúde são os óbitos maternos, que podem ser obstétricos diretos ou indiretos, sendo os diretos resultantes de complicações presentes durante a gravidez, parto e pós-parto, enquanto que os óbitos indiretos são decorrentes de patologias preexistentes ou que surgiram durante a gestação e foram acentuadas pelos efeitos fisiológicos do período gestacional (BRASIL MS, 2012).

Para tanto, é importante que as consultas do pré-natal sejam iniciadas precocemente, até a $12^{a}$ semana de gestação (LIVRAMENTO DVP et al., 2018).

Geralmente isso pode não ocorrer devido alguns obstáculos que vão desde fatores voltados ao acesso das gestantes à assistência obstétrica, tais como, distribuição geográfica dos serviços de saúde, local de moradia das referidas usuárias, mães em situação de pobreza (SAMPAIO AF et al., 2018), às dificuldades assistenciais, como por exemplo: consulta clínica inadequada, profissionais despreparados, falhas na graduação associada a inexperiência do recém-formado, baixas qualificações, ausência de cursos de capacitação profissional, dentre outros (VIELLAS EF et al, 2014; RAMOS ASMS et al., 2018).

Por outro lado, sabemos que as ações do enfermeiro na atenção primária de saúde sobrepõe o modelo biomédico, hospitalocêntrico, hegemônico no Brasil ao proverem práticas de cuidado seguras, eficientes e de alta qualidade, que fortalecem os serviços de atenção básica, trazendo uma visão ampliada e integral das necessidades e das diversas situações envolvendo o processo saúde-doença do indivíduo, família e comunidade (STARFIELD B, 2012; FERTONANI HP et al, 2015).

Outras atribuições realizadas pelo enfermeiro, além de reorganizar o modelo de saúde, proposto pela Estratégia de Saúde da Família (ESF), que vão desde a organização gerencial das equipes de saúde ao funcionamento e cuidados diretos a população adscrita (CAÇADOR BS et al., 2015).

De todas essas atividades exercidas pelo enfermeiro, cabe ressaltar a gestão do cuidado clínico a gestante, caracterizada pela consulta de enfermagem no pré-natal, que oportuniza conhecê-la, através da interação desta com o profissional (POHLMANN FC et al, 2016), otimizando o desenvolvimento de uma prática clínica voltada a escuta qualificada, avaliação das condições de saúde biopsicossociais, espirituais e prestação do cuidado integral a mulher gestante e ao concepto.

Neste sentido a consulta de enfermagem às gestantes deve oferecer assistência integral, clínicoginecológica e educativa, visando o controle do pré-natal, parto, puerpério e nascimento saudável (BARBOSA TLA et al., 2011).

Ainda segundo Barbosa TLA (2011) na assistência pré-natal cabe ao enfermeiro mostrar à população a importância do acompanhamento da gestação na promoção da saúde, prevenção e tratamento de distúrbios, durante e após a gravidez e, informá-la dos serviços disponíveis.

Essas ações contribuem para uma maior adesão das mulheres ao pré-natal, tornando-as mais partícipe, o que, em última análise, será essencial para redução dos elevados índices e mortalidade materna e perinatal verificados no Brasil nos últimos anos (VIANA GL, FREITAS EAM, 2016; ERICO LSP et al, 2018). 
Tendo em vista a característica da ESF, espera-se que o enfermeiro ao realizar as ações acima descritas e particularmente no que diz respeito ao pré-natal, seja capaz de identificar o mais previamente possível os fatores determinantes ou condicionantes relacionados aos riscos e agravos à saúde da mulher (VALENÇA CN, 2010), mas para isso, faz-se necessário um número mínimo de consultas: seis, preconizado pelo Ministério da Saúde (MINISTÉRIO DA SAÚDE BR, 2007; PIO DAM, OLIVEIRA MM, 2014) e seu sucesso depende em grande parte do momento em que se inicia o atendimento anteparto, considerado condição sine qua non, ou seja, essencial e indispensável para o controle dos índices de morbimortalidade materna-neonatal (NOGUEIRA LDP, OLIVEIRA GS, 2017).

Justifica-se este estudo por tornar-se importante devido à relevância na realização de um adequado prénatal para prevenção de complicações durante o período gestacional, o parto e o puerpério para a redução dos indicadores de saúde, tendo como objetivo do trabalho: avaliar o impacto do indicador de morbimortalidade materno-neonatal, através das dificuldades vivenciadas por enfermeiros no atendimento ao pré-natal de risco habitual.

\section{METODOLOGIA}

Trata-se de um estudo de revisão integrativa da literatura, com abordagem quantitativa e qualitativa sobre pré-natal de risco habitual.

No geral, para a construção da revisão integrativa é preciso percorrer seis etapas distintas, similares aos estágios de desenvolvimento de pesquisa convencional, a saber, identificação do tema e seleção da hipótese ou questão de pesquisa para a elaboração da revisão integrativa, estabelecimento de critérios para inclusão e exclusão de estudos/ amostragem ou busca na literatura, definição das informações a serem extraídas dos estudos selecionados/ categorização dos estudos, avaliação dos estudos incluídos na revisão integrativa, interpretação dos resultados e apresentação da revisão/síntese do conhecimento (MENDES KDS et al., 2018).

O levantamento bibliográfico realizado pelos (as) autores (as) foi feito por meio da consulta direta pela internet, no endereço eletrônico da plataforma da Biblioteca Virtual de Saúde (BVS), nas seguintes bases de dados: literatura latino-americana e do caribe em ciências da saúde (LILACS), medical literature analysis and retrieval system online (MEDLINE) via PUBMED, Scientific Electronic Library Online (SCIELO) e Centro Latino-Americano e do Caribe de Informação em Ciências da Saúde (BIREME).

Foi utilizado o software EndNote para gerenciar as referências e importar os artigos das bases de dados, organizando os artigos em dois grupos. O primeiro grupo foi chamado de: "selecionados para leitura na íntegra" e o segundo grupo, foi chamado de: "excluídos após leitura de título, resumo e artigo completo.

Foram incluídos os artigos originais, disponíveis na íntegra, que apresentaram uma abordaram quantitativa e qualitativa a temática, quanto as principais dificuldades encontradas pelos enfermeiros de uma ESF, voltados ao atendimento de pré-natal da gestante em situação de risco habitual e sua relação com os indicadores de morbimortalidade materno-neonatal, sem restrição de idiomas ou país de publicação, além da seleção dos artigos publicados dentro de um recorte temporal de 2006 a 2018, disponíveis nas bases de dados eletrônicos da Lilacs, Scielo, Bireme e Medline via Pubmed e por outras fonte (SCORPUS).

Para critérios de exclusão, foram desconsiderados os artigos que fugiram do tema proposto pelos autores, que se encontravam duplicados nas bases de dados eletrônicas, manuais técnicos, revisão de literatura, exceto revisão sistemática devido seu alto grau de evidência.

Para a seleção dos artigos, fez-se necessário que os autores respeitassem os critérios de inclusão e exclusão, caso gerasse alguma dúvida quanto ao objeto de estudo, foi preciso que os pesquisadores realizassem releitura dos títulos, em caso dos títulos não comportassem as informações suficientes, se realizou uma releitura dos resumos e descritores e se mesmo assim houvesse alguma incerteza sobre sua inclusão, se realizou a leitura completa dos artigos e mesmo diante de todas essas etapas percorridas, se por ventura ainda permanecesse a dúvida, se optou pela escolha de três revisores, para segunda e última análise. 
Por sua vez, os três revisores de forma independente refez todo percurso metodológico realizado anteriormente pelos pesquisadores e de maneira criteriosa, com rigor técnico e pleno conhecimento dos critérios previamente estabelecidos e mesmo após todas as etapas criteriosamente percorridas, em caso de dúvida quanto a definição sobre a inclusão dos artigos para composição da amostra final, se utilizou a técnica de consenso.

A seleção dos artigos foi efetuada nos meses de agosto de 2018 a fevereiro de 2019, uma consulta aos Descritores em Ciencias da Saude (DeCS) proprios as bases de dados LILACS, SciELO e BIREME e por meio dos descritores cadastrados no Medical Subject Headings (MeSH) próprio da base de dados PubMed, para isso, foi preciso determinar os termos livres de busca (MeSH TERMS), para então efetuar o cruzamento entre eles, utilizando os operadores booleanos "AND" exemplificados na tabela 1.

Tabela 1 - Estratégias de busca para consulta em bases de dados eletrônicas.

\begin{tabular}{|c|c|c|}
\hline Cruzamento em inglês & Cruzamento em espanhol & Cruzamento em português \\
\hline $\begin{array}{l}\text { Gravitidies (Decs) and } \\
\text { Maternal-child } \\
\text { nursing (Mesh Terms). }\end{array}$ & $\begin{array}{l}\text { Mujeres Embarazadas (Decs) } \\
\text { and Enfermería Materno } \\
\text { infantil (Mesh Terms). }\end{array}$ & $\begin{array}{l}\text { Gestantes (Decs) and } \\
\text { Enfermagem Materno-Infantil } \\
\text { (Mesh Terms). }\end{array}$ \\
\hline $\begin{array}{l}\text { Gravitidies (Decs) and } \\
\text { Primary Health Care } \\
\text { (Mesh Terms). }\end{array}$ & $\begin{array}{l}\text { Mujeres Embarazadas (Decs) } \\
\text { Atención Primaria de } \\
\text { Salud (Mesh Terms). }\end{array}$ & $\begin{array}{l}\text { Gestantes (Decs) and } \\
\text { Atenção básica (Mesh } \\
\text { Terms). }\end{array}$ \\
\hline $\begin{array}{l}\text { Gravitidies (Decs) and } \\
\text { Quality of Health Care } \\
\text { (Mesh Terms). }\end{array}$ & $\begin{array}{l}\text { Mujeres Embarazadas (Decs) and } \\
\text { Calidad de la Atención de Salud } \\
\text { (Mesh Terms). }\end{array}$ & $\begin{array}{l}\text { Gestantes (Decs) and } \\
\text { qualidade da assistência à } \\
\text { saúde (Mesh Terms). }\end{array}$ \\
\hline $\begin{array}{l}\text { Live Birth (Decs) and } \\
\text { Maternal-child } \\
\text { nursing (Mesh Terms). }\end{array}$ & $\begin{array}{l}\text { Nacimiento Vivo (Decs) and } \\
\text { Enfermería Materno-infantil (Mesh } \\
\text { Terms). }\end{array}$ & $\begin{array}{l}\text { Nascimento Vivo (Desc) and } \\
\text { Enfermagem materno-infantil } \\
\text { (Mesh Terms). }\end{array}$ \\
\hline
\end{tabular}

Fonte: bases de dados da LILACS, SCIELO, BIREME e MEDLINE via PUBMED.

\section{RESULTADOS}

Inicialmente foram identificados um total de 1.537 artigos provenientes de periódicos internacionais (61\%) e nacionais (39\%).

Para se ter um panorama mais abrangente sobre o tema, se utilizou também outra fonte de banco de dados eletrônicos (SCORPUS).

Foi também excluídos os artigos repetidos, tais como, LILACS (149); SCIELLO (143); BIREME (144); PUBMED (66); MEDLINE via PUBMED (63); SCORPUS (4), totalizando 569 artigos duplicados.

Além daqueles eliminados por critérios de exclusão e de elegibilidade, obtendo-se, portanto, uma amostra final de cinco estudos $(n=5)$ referente ao tema em estudo. A estratégia utilizada para seleção dos artigos está representada na Figura 1. 
Figura 1 - Fluxograma com estratégias de seleção dos artigos, após busca em base de dados eletrônicos, $2006-2018$.

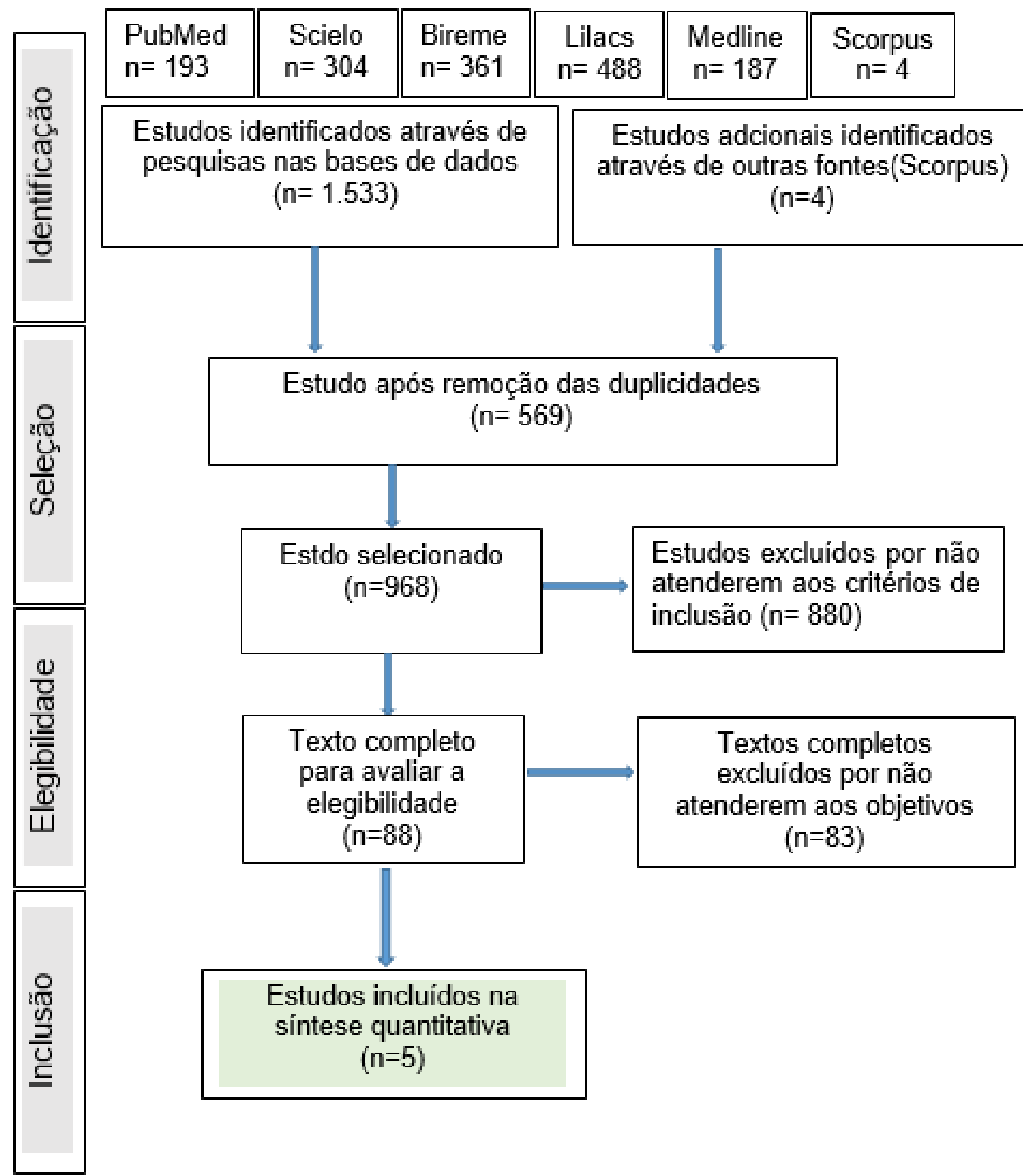

Fonte: Fluxograma construída pelos (as) autores (as), utilizando como fonte as bases de dados do LILACS, SCIELO, BIREME e MEDLINE via PUBMED.

A Tabela 2 mostra a descrição da análise dos artigos finais $(n=5)$ que se enquadram no espaço temporal de 2006 a 2018, subdivididos por autor/ano, desenho metodológico, objetivo, principais resultados e conclusão. Quanto ao desenho metodológico, foram selecionados três artigos de abordagem quantitativa e dois qualitativa. 
Tabela 2 - Análise dos artigos quanto as dificuldades dos enfermeiros de uma ESF no atendimento de pré-natal de risco habitual, 2006 a 2018.

\begin{tabular}{llll}
\hline Autores/ano & Metodologia & Objetivo & Resultados
\end{tabular}

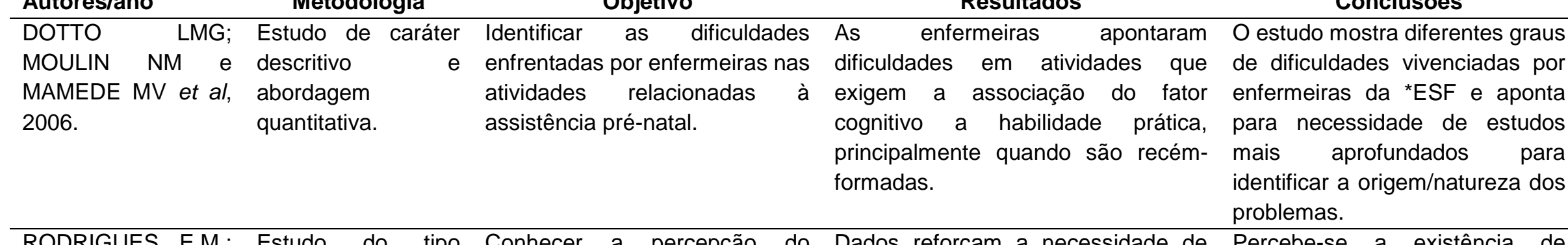

\begin{tabular}{|c|c|c|c|c|}
\hline $\begin{array}{l}\text { RODRIGUES E.M.; } \\
\text { NASCIMENTO R.G.; } \\
\text { ARAÚJO A, } 2011 .\end{array}$ & $\begin{array}{l}\text { Estudo do tipo } \\
\text { qualitativo, } \\
\text { envolvendo } \\
\text { enfermeiros de uma } \\
{ }^{*} \text { ESF. }\end{array}$ & $\begin{array}{l}\text { Conhecer a percepção do } \\
\text { enfermeiro acerca do uso do } \\
\text { protocolo de suas atribuições na } \\
\text { assistência pré-natal, focando } \\
\text { pontos dificultadores. }\end{array}$ & $\begin{array}{l}\text { Dados reforçam a necessidade de } \\
\text { investimentos na formação de } \\
\text { enfermeiros para atendimento à } \\
\text { mulher em todo ciclo gravídico- } \\
\text { puerperal, com criação de protocolos. }\end{array}$ & $\begin{array}{l}\text { Percebe-se a existência de } \\
\text { lacunas na assistência pré-natal } \\
\text { e uma forte resistência da gestão } \\
\text { em organizar a atenção básica } \\
\text { para a assistência a gestante. }\end{array}$ \\
\hline $\begin{array}{l}\text { FONTANELLA APS; } \\
\text { WISNIEWSKI D, } \\
2014 .\end{array}$ & $\begin{array}{lr}\text { Estudo do tipo } \\
\text { transversal, } & \text { de } \\
\text { abordagem } & \\
\text { quantitativa. } & \end{array}$ & $\begin{array}{l}\text { Identificar as dificuldades dos } \\
\text { enfermeiros para realização da } \\
\text { consulta de pré-natal à gestante } \\
\text { de baixo risco, numa }{ }^{* *} \mathrm{ABS} \\
\text { município do interior do Paraná. }\end{array}$ & $\begin{array}{l}44,4 \% \text { dos enfermeiros relataram } \\
\text { haver dificuldades para realizar o pré- } \\
\text { natal na }{ }^{* *} A B S \text {, que vão desde as } \\
\text { condições inadequadas do ambiente } \\
\text { a baixas capacitações do enfermeiro. }\end{array}$ & $\begin{array}{l}\text { As dificuldades são consideradas } \\
\text { relevantes e podem influenciar no } \\
\text { desfecho materno e neonatal, } \\
\text { além de prejudicar o atendimento } \\
\text { de pré-natal adequado e/ou } \\
\text { qualificado. }\end{array}$ \\
\hline $\begin{array}{l}\text { TOMASI E; FASSA } \\
\text { AG, 2017. }\end{array}$ & $\begin{array}{l}\text { Estudo do } \\
\text { descritivo, } \\
\text { quantitativo } \\
\text { documental. }\end{array}$ & $\begin{array}{l}\text { Descrever indicadores de } \\
\text { qualidade da atenção pré-natal } \\
\text { no Brasil no âmbito do ***PMAQ- } \\
\text { AB. }\end{array}$ & $\begin{array}{l}\text { Apenas } 15 \% \text { das entrevistadas } \\
\text { receberam adequada atenção de pré- } \\
\text { natal e menos de } 1 / 4 \text { das gestantes } \\
\text { referiram ter recebido todos os } \\
\text { procedimentos. }\end{array}$ & $\begin{array}{l}\text { Em favor da redução das } \\
\text { desigualdades, sugere-se que o } \\
\text { tema da qualidade da atenção } \\
\text { pré-natal na }{ }^{* \star *} \text { RBS, continue a } \\
\text { ser estudado. }\end{array}$ \\
\hline $\begin{array}{l}\text { LEAL } \\
\text { BARREIRO MSC; } \\
\text { MENDES RB et al, } \\
2018 .\end{array}$ & $\begin{array}{l}\text { Estudo do } \\
\text { descritivo } \\
\text { qualitativo. }\end{array}$ & $\begin{array}{l}\text { Avaliar a assistência ao pré- } \\
\text { natal de baixo risco pelo } \\
\text { enfermeiro de Lagarto- Sergipe. }\end{array}$ & $\begin{array}{l}\text { Embora seja satisfatório o pré-natal } \\
\text { realizado por enfermeiros, ainda há } \\
\text { necessidades de novas estratégias } \\
\text { na atenção básica para melhorar o } \\
\text { atendimento. }\end{array}$ & $\begin{array}{l}\text { Há necessidade de qualificações } \\
\text { para enfermeiros que atuam no } \\
\text { pré-natal, visando reduzir os } \\
\text { riscos de morbimortalidade nas } \\
\text { gestantes. }\end{array}$ \\
\hline
\end{tabular}

FONTE: Tabela elaborada pelos (as) autores (as) do projeto, utilizando como fonte as bases de dados LILACS, SCIELO, BIREME e MEDLINE, via PUBMED. *Estratégia de Saúde da Família; **Atenção Básica de Saúde; *** Programa de Melhoria do Acesso e da Qualidade; ${ }^{* * \star *}$ Rede básica de saúde.

REAS/EJCH | Vol.Sup.20 | e711 | DOI: https://doi.org/10.25248/reas.e711.2019 Página 7 de 11 


\section{DISCUSSÃO}

De acordo com o Ministério da Saúde, no Brasil observa-se uma cobertura crescente da assistência prénatal, que em 1994 era abaixo de $90 \%$ e se intensificou a partir de 2011, com a implantação da Rede Cegonha e do Programa Nacional de Melhoria do Acesso e da Qualidade da Atenção Básica (PMAQ-AB), alcançando valores superiores a $97 \%$ em todas as regiões do país e em mulheres com diferentes características demográficas, sociais e reprodutivas, por ampliar o acesso dessas usuárias a atenção primária de saúde (ROSA CQ et al., 2014).

Contudo, mesmo diante desses avanços, a adequação dessa assistência é ainda baixa, pois $43 \%$ das

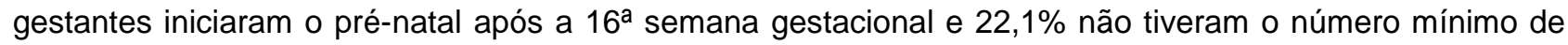
consultas previstas para a idade gestacional (GURGEL RQ et al, 2018), o que indica busca tardia desta assistência e número insuficiente de atendimentos, situações também observadas em outros estudos nacionais (PRADO DS et al, 2018).

Entretanto, mesmo com as melhorias no acesso à assistência pré-natal, dados revelam que os índices de mortalidade materno-infantil ainda são preocupantes, especialmente os relacionados às causas ginecoobstétricas (DOMINGUES RMS, 2015).

Esta realidade denota um problema alarmante e, portanto, requer intervenção imediata e eficaz, posto que o estado de saúde da mãe tem relevância direta para o bebê ainda em formação (ANDERSON MS e HAY WW, 2017).

Segundo, a Organização Mundial da Saúde (OMS) define morte materna (MM) aquela que acontece durante a gravidez ou 42 dias após o término da gravidez, por qualquer causa relacionada à gestação ou ao seu manejo, sendo considerada um grave problema de saúde pública, e é evidência do nível de desenvolvimento de uma determinada população ou região (OMS, 2016). Aproximadamente $99 \%$ de todas as MMs ocorrem em países em desenvolvimento (SAY L et al., 2014).

A maioria dessas mortes poderia ser evitada se os sistemas de saúde garantissem às mulheres acesso a serviços de alta qualidade e ampliação da cobertura pré-natal em toda sua totalidade (ROSA CQ et al., 2014). No Brasil, a estimativa da taxa de mortalidade materna deveria ser menor ou igual a 33 mortes por 100.000 nascidos vivos até 2020 (BRASIL MS, 2014), mas esse objetivo está longe de ser cumprido.

Para Tomasi E et al. (2017), uma atenção pré-natal de qualidade é capaz de diminuir a morbidade e a mortalidade materno-infantil, uma vez que a identificação do risco gestacional pelo profissional de enfermagem permite a orientação e os encaminhamentos adequados em cada momento da gravidez. Entretanto para Leal NJ et al. (2018), outro impasse é a ausência de contra referência, o que prejudica o acompanhamento contínuo dessa gestante em todos os níveis de assistência.

As dificuldades assistenciais do enfermeiro no pré-natal foram mencionadas por Dotto LMG et al. (2006) e colaboradores desde 2006 e foram classificadas como dificuldades moderadas a muita dificuldade. Em relação a condição moderada, um dos problemas relevantes que podemos citar é a realização de visita domiciliar em uma área territorial grande, quando esta é adscrita a uma ESF.

Entretanto em outro estudo, a visita domiciliar não foi motivo de dificuldade para os enfermeiros, por ser uma atividade que merece ser reforçada, pois é o momento de buscar as gestantes faltosas, além do trabalho educativo voltado ao fortalecimento de vínculos entre ela e seu grupo familiar (SCHIMER J, 2012).

Outros problemas também descrito por Dotto LMG et al. (2006) considerados como moderados foram as prescrições de medicamentos, o exame físico específico gineco-obstétrico no lugar do exame físico completo e detalhado em virtude da grande demanda de gestantes, em torno de 250 consultas ao mês e a identificação de fatores de risco, este último também enfatizado por Tomasi $E$ et al. (2017) doze anos depois.

Já em relação as consideradas de muita dificuldade podemos citar, a solicitação e avaliação de exames laboratoriais, além da demora do laboratório em fornecer o resultado do exame, com tempo mínimo em média de três meses e também para marcação de outros (DOTTO LMG et al., 2006; PEREIRA KGP et al., 2015). 
Um aspecto que chama atenção quanto as dificuldades no atendimento dos enfermeiros ao pré-natal, é a condição de trabalho, a estrutura física dos serviços, a disponibilidade dos equipamentos para investigação clínica-gineco-obstétrica de qualidade, além da organização da demanda para atendimento as gestantes, isso porque o enfermeiro em alguns momentos exerce mais funções administrativas que assistencial (SILVA ALS et al., 2015).

Outro problema é que a maioria dos estudos publicados aponta para dificuldades na captação precoce das gestantes que deve ocorrer em até 120 dias da gestação (DARMONT MQR et al., 2010; BRASIL MS, 2014; MARTINS PQM et al., 2015).

Além da não adesão das mesmas ao pré-natal, que pode ser reflexo tanto da falta de informação ou insatisfação, quanto a falta de acolhimento disponibilizados por alguns enfermeiros, ou até mesmo por fatores socioeconômicos da mãe, tais como, baixa renda familiar e escolaridade, local de residência distante do serviço e custo para o deslocamento; da qualidade dos cuidados em saúde e de suporte social (LEAL NJ et al., 2018).

Sabe-se que o acolhimento de qualidade contribui bastante para a adesão e o vínculo das gestantes ao serviço de pré-natal, por ser pautado nas relações de interação, confiança, capacidade de escuta, relações horizontais e terapêuticas resolutivas, entre as enfermeiras e essa população (AGUIAR RS et al., 2013). Entretanto, outros estudos enfatizam sobre a redução do tempo de espera no atendimento, que também seria importante para promover um ambiente acolhedor e de acesso facilitado a essa atenção de saúde (GUERREIRO EM et al., 2012; SILVA ZN et al., 2014).

Outros fatores potencialmente relacionados as dificuldades encontradas pelos enfermeiros quanto ao atendimento das gestantes na atenção primária é a baixa adesão ao pré-natal por mulheres com idades extremas, a não convivência dessas mães com o companheiro, uso de álcool ou drogas na gravidez, multiparidade, a não aceitação da gestação, a falta de apoio familiar, o contexto social adverso, as experiências negativas de atendimento e as concepções equivocadas de descrédito sobre 0 pré-natal (DARMONT MQR et al., 2010; MARTINS PQM et al., 2015).

No tocante a qualidade dos serviços de saúde, Ximenes et al. (2008) e colaboradores afirmam que o indicador de assistência à saúde gestacional pode implicar na ocorrência de várias circunstâncias especificas de risco, não podendo ser determinada por um único fator.

Outras pesquisas semelhantes ao estudo supracitado também enfatizam que existem vários fatores determinantes que interferem na qualidade da atenção à saúde gestacional, tais como, as dificuldades de acesso, a baixa qualidade da atenção ao pré-natal, que somados a esses indicadores, se tem a exclusão prénatal, aumentando assim a possibilidade de gestações prematuras, retardo do crescimento intrauterino, baixo peso ao nascer e óbitos maternos e infantis por afecções no período peri e pós-natal (COSTA JSD, 2014).

Outros fatores determinantes que interferem na qualidade da atenção à saúde materna é o déficit na ampliação dos serviços de pré-natal e na sua cobertura em algumas regiões do país. Essas dificuldades decorrem, principalmente, da falta de recursos humanos e materiais, entre outros, acarretando sérios obstáculos à implantação de ações de enfermagem embasadas por princípios de qualidade nos diversos serviços de atenção à saúde da mulher, ocasionando sobrecarga de atividades (GUERREIRO EM et al., 2012).

A melhoria da assistência pré-natal não está pautada apenas no atendimento clínico em si, mas na integração entre os diferentes serviços de saúde necessários à mãe e ao neonato, vinculados em Redes de Atenção à Saúde, assegurando o acesso oportuno a serviços de qualidade (MIRANDA CS et al., 2017). Dentro dessa perspectiva, outros estudos enfatizaram que a adesão ao pré-natal deve ser estimulada pelos enfermeiros nas consultas pré-concepcionais e nas visitas domiciliares para a detecção precoce de gestação (GOMES CBA et al., 2019). O conhecimento das ações de pré-natal é relevante para o entendimento dos principais avanços alcançados e dos obstáculos a serem superados pelo Sistema Único de Saúde (SUS), rumo a melhoria dos indicadores de saúde materna. 


\section{CONSIDERAÇÕES FINAIS}

Os dados permitem compreender que o acesso ao pré-natal é adequado em grande parte das situações, porém em outras não, devido ao surgimento de algumas barreiras enfrentadas tanto pelas gestantes que vão desde as iniquidades sociais por elas vivenciadas, as dificuldades de acessibilidade a esses serviços de saúde, como também por profissionais no atendimento integral a essa população específica, havendo necessidade de estratégias eficazes para a melhoria do cuidado as mulheres em situação de gestação e assim diminuir a ocorrência de desfechos fatais, como a mortalidade materna-neonatal. Torna-se evidente a necessidade de implementação de políticas públicas voltadas à saúde da mulher, objetivando o atendimento absoluto a essas mães, implantando medidas que vão desde a captação precoce da gestação, prevenção de riscos e promoção à saúde.

\section{REFERÊNCIAS}

1. AGUIAR RS, et al. Perception of women about the nurse's reception in prenatal care. Revista Cogitare Enfermagem, 2013; 18(4): 756-60.

2. ALMEIDA CAL, TANAKA OY. Perspectiva das mulheres na avaliação do Programa de Humanização do Pré-Natal e Nascimento. Revista de saúde pública, 2009; 43(1): 98-104.

3. ANDERSON MS, HAY WW. Restrição do crescimento intra-uterino e o recém-nascido pequeno para idade gestacional. Avery neonatologia fisiopatologia e tratamento do recém-nascido. 8nd ed. Rio de Janeiro: Guanabara Koogan, 2017; 448-478.

4. BARBOSA TLA, et al. O pré-natal realizado pelo enfermeiro: a satisfação das gestantes. Revista Cogitare Enfermagem, 2011; 16(1): 29-35.

5. BEYEA SC, NICOLL LH. Writing an integrative review. AORN J. 1998; 67(4): 877-80.

6. BIBHA SER, TEIJLINGEN MPPS. Factors affecting the utilization of antenatal care in developing countries: systematic review of the literature, 2008; 61(3): 244-60.

7. BRASIL. Ministério da Saúde. SECRETARIA DE ATENÇÃO À SAÚDE. DEPARTAMENTO DE AÇÕES PROGRAMÁTICAS ESTRATÉGICAS. Política nacional de atenção integral à saúde da mulher: princípios e diretrizes / Ministério da Saúde, Secretaria de Atenção à Saúde, Departamento de Ações Programáticas Estratégicas. Brasília: Ministério da Saúde, 2004.

8. BRASIL. Ministério da Saúde. Gestação de alto Risco Manual Técnico. Brasília: Ministério da Saúde, 2010.

9. BRASIL. Ministério da Saúde. Secretaria de Atenção à Saúde. Departamento de Atenção Básica. Atenção ao prénatal de baixo risco / Ministério da Saúde. Secretaria de Atenção à Saúde. Departamento de Atenção Básica. Brasília: Ministério da Saúde, 2012.

10. BRASIL. Ministério da Saúde. Portaria 1.459/2011 de 24 de junho de 2011. Institui, no âmbito do Sistema Único de Saúde - SUS - a Rede Cegonha. Brasília: Ministério da Saúde, 2011.

11. BRASIL. Ministério da Saúde. Secretaria de Atenção à Saúde, Departamento de Atenção Básica. Atenção Básica e Saúde da Família. Brasília: Ministério da Saúde, 2014.

12. CAÇADOR BS, et al. Ser enfermeiro na estratégia de saúde da família: desafios e possibilidades. REME - Revista Mineira de Enfermagem, 2015; 3(19).

13. COSTA CSC et al. Características do atendimento pré-natal na Rede Básica de Saúde. Revista Eletrônica de Enfermagem, 2016; 15(2): 516-522.

14. COSTA JSD et al. Cuidados de enfermagem no pré-natal e segurança do paciente: revisão integrativa. Revista Enfermagem UFPE online, 2014; (10): 4909-4919.

15. DARMONT MQR et al. Adesão ao pré-natal de mulheres HIV+ que não fizeram profilaxia da transmissão vertical: um estudo sócio-comportamental e de acesso ao sistema de saúde. Caderneta Saude Pública, 2010; 26(9): 178896.

16. DOMINGUES RMSM et al. Adequação da assistência pré-natal segundo as características maternas no Brasil. Revista Panam Salud Publica, 2015; 37(3): 140-7.

17. DOTTO LMG et al. Prenatal care: difficulties experienced by nurses. Revista Latino-Americana Enfermagem, 2006; 14(5): 682-8.

18. DUARTE SJH, Almeida EP DE. O papel do enfermeiro doprograma saúde da família no atendimento pré-natal. Revista de enfermagem, 2014; 1029-1035.

19. FERTONANI HP et al. The health care model: concepts and challenges for primary health care in Brazil. Ciência Saúde Coletiva, 2015; 20(6): 1869-78. 
20. FONTANELLA APS, WISNIEWSKI D. Pré-natal de baixo risco: Dificuldades encontradas pelos profissionais enfermeiros. Brazilian Journal of Surgery and Clinical Research - BJSCR, 2014; 7(3): 11-16.

21. GAMA SGN et al. Fatores associados à assistência pré-natal precária em uma amostra de puérperas adolescentes em maternidades do Município do Rio de Janeiro, 2004; 20(1): 101-11.

22. GOMES CBA et al. Consulta de enfermagem no pré-natal: narrativas de gestantes e enfermeiras, 2019; $28: 1$-15.

23. GUERREIRO EM et al. O cuidado pré-natal na atenção básica de saúde sob o olhar de gestantes e enfermeiros. REME - Revista Mineira de Enfermagem, 2012; 16(3): 1-6.

24. LEAL NJ et al. Assistência ao pré-natal: depoimento das enfermeiras. Revista Cuidado é Fundamental, 2018; 10(1): 113-122.

25. LIVRAMENTO DVP et al. Percepção das gestantes acerca do cuidado no pré-natal na atenção primária à saúde. Revista Gaúcha Enfermagem, 2019; (40): 1-10.

26. MENDES KDS et al. Revisao Integrativa: metodo de pesquisa para a incorporacao de evidencias na saude e na enfermagem, 2008; 17(4): 758-64.

27. MIRANDA MHH et al. Determinantes associados à mortalidade perinatal e fatores associados. Revista de enfermagem UFPE onlina, 2017; 11(3): 1171-8.

28. NOGUEIRA LDP; OLIVEIRA GS. Assistência pré-natal qualificada: as atribuições do enfermeiro - um levantamento bibliográfico. Revista de Enfermagem e Atenção Saúde, 2017; 6(1): 107-119.

29. PIO DAM; OLIVEIRA MM. Educação em saúde para atenção à gestante: paralelo de experiências entre Brasil e Portugal. Saúde e Sociedade, 2014; 23(1): 313-24.

30. POHLMANN FC et al. Prenatal care model in the far south of Brazil. 2016; 25(1): 1-8.

31. RAMOS ASMS et al. Assistência de enfermagem no pré-natal de baixo risco na atenção primária. J Manag Prim Health Care, 2018.

32. RODRIGUES EM et al. Protocolo de assistência de pré-natal ações, facilidades e dificuldades dos enfermeiros da estratégia de saúde da família. Revista Escola de Enfermagem da USP, 2011; 45(5): 1041-1047.

33. ROSA CQ et al. Factors associated with lack of prenatal care in a large municipality. Revista Saúde Pública, 2014; 48(6): $977-84$.

34. SAY L et al. Global causes of maternal death: a WHO systematic analysis. Lancet Glob Health, 2014; 2(6): 323333.

35. SILVA ALS et al. Práticas de enfermeiras para promoção da dignificação, participação e autonomia de mulheres no parto normal. Escola Anna Nery Revista de Enfermagem, 2015; 19(3): 424-31.

36. STARFIELD B. Primary care: an increasingly important contributor to effectiveness, equity, and efficiency of health services, 2012; (1): 20-6.

37. TOMASI E et al. Qualidades de atenção ao pré-natal na rede básica de saúde no Brasil: Indicadores e desigualdades sociais. Caderneta Saúde Pública, 2017; 33(3).

38. VALENÇA CN, GERMANO RM. Prevenindo a Depressão Puerperal na Estratégia Saúde da Família: Ações do Enfermeiro no Pré-Natal. Rev Rene, 2010; 11(2): 129 - 139.

39. VIELLAS EF et al. Assistência pré-natal no Brasil. Caderneta Saúde Pública, 2014; 30(1): 85-100.

40. XIMENES NFRG et al. Qualidade da atenção ao pré-natal na Estratégia Saúde da Família em Sobral. Revista Brasileira de Enfermagem, 2008; 61(5): 595-602. 